\title{
Vitrification and nanocrystallization of pure liquid Ni studied using molecular-dynamics simulation
}

Cite as: J. Chem. Phys. 151, 124502 (2019); https://doi.org/10.1063/1.5119307

Submitted: 12 July 2019 . Accepted: 03 September 2019. Published Online: 24 September 2019

D. V. Louzguine-Luzgin (D), M. Miyama, K. Nishio, A. A. Tsarkov (D), and A. L. Greer (D)
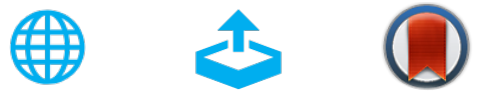

\section{ARTICLES YOU MAY BE INTERESTED IN}

Configurational entropy of glass-forming liquids

The Journal of Chemical Physics 150, 160902 (2019); https://doi.org/10.1063/1.5091961

Does the Adam-Gibbs relation hold in simulated supercooled liquids?

The Journal of Chemical Physics 151, 084504 (2019); https://doi.org/10.1063/1.5113477

Universal nature of dynamic heterogeneity in glass-forming liquids: A comparative study of metallic and polymeric glass-forming liquids

The Journal of Chemical Physics 151, 184503 (2019); https://doi.org/10.1063/1.5125641

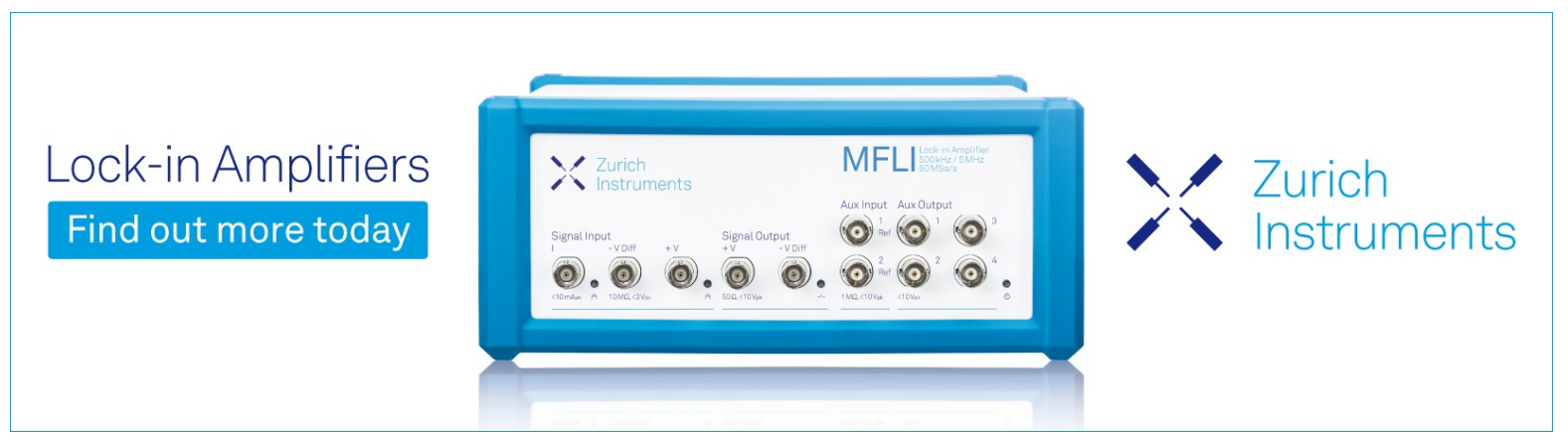




\title{
Vitrification and nanocrystallization of pure liquid Ni studied using molecular-dynamics simulation
}

\author{
Cite as: J. Chem. Phys. 151, 124502 (2019); doi: 10.1063/1.51 19307 \\ Submitted: 12 July 2019 Accepted: 3 September 2019 • \\ Published Online: 24 September 2019
}

D. V. Louzguine-Luzgin, ${ }^{1,2, a)}$ (D) M. Miyama, ${ }^{3}$ K. Nishio, ${ }^{1,4}$ A. A. Tsarkov, ${ }^{5}$ (D) and A. L. Greer ${ }^{2,6}$ (D)

\begin{abstract}
AFFILIATIONS
${ }^{1}$ MathAM-OIL, National Institute of Advanced Industrial Science and Technology (AIST), Sendai 980-8577, Japan

${ }^{2}$ WPI Advanced Institute for Materials Research, Tohoku University, Aoba-Ku, Sendai 980-8577, Japan

${ }^{3}$ Department of Applied Information Sciences, Graduate School of Information Sciences, Tohoku University, Sendai 980-8579, Japan

${ }^{4}$ Research Center for Computational Design of Advanced Functional Materials (CD-FMAT), National Institute of Advanced Industrial Science and Technology (AIST), Central 2, Umezono 1-1-1, Tsukuba, Ibaraki 305-8568, Japan

${ }^{5}$ National University of Science and Technology "MISiS", Moscow 119049, Russia

${ }^{6}$ Department of Materials Science and Metallurgy, University of Cambridge, Cambridge CB3 OFS, United Kingdom
\end{abstract}

a) Author to whom correspondence should be addressed: dml@wpi-aimr.tohoku.ac.jp. Tel.: +081-22-217-5957.

Fax: +081-22-217-5957.

\begin{abstract}
Structural variation, vitrification, and crystallization processes in liquid nickel are simulated on continuous cooling and isothermal holding using a classical molecular-dynamics computer simulation procedure with an embedded-atom method potential at constant pressure. Structural changes are monitored with direct structure observation in the simulation cells, as well as by pair distribution and radial distribution functions created using the atomic coordinates. A cluster analysis is also performed. The crystallization kinetics is analyzed under isothermal conditions by monitoring density and energy variation as a function of time. As a result, a time-temperature-transformation diagram can be constructed over a wide temperature range.
\end{abstract}

Published under license by AIP Publishing. https://doi.org/10.1063/1.5119307

\section{INTRODUCTION}

For kinetic reasons that are related to difficulties in the crystal nucleation and growth processes at lower temperatures, it is possible to undercool/supercool ${ }^{1,2}$ a liquid below the liquidus temperature $\left(T_{1}\right)$ and form a disordered glassy phase. ${ }^{3,4}$ This vitrification of supercooled liquids has been studied in detail through experiments, ${ }^{5,6}$ theoretical studies, ${ }^{7,8}$ and computer simulation. 9, The studies were performed for metallic glasses, ${ }^{11,12}$ including pure metals ${ }^{13,14}$ and other substances. ${ }^{15}$

The structure of liquids and glasses has also been studied extensively ${ }^{17,18}$ including in situ methods using synchrotron-radiation $\mathrm{X}$-ray diffractometry, ${ }^{19,20}$ neutron diffraction, ${ }^{21,22}$ and transmission electron microscopy. ${ }^{23,24}$ Both reciprocal and real-space distribution functions were used for modeling thermal expansion of a glassy alloy. ${ }^{25}$ However, many (but not all $^{26}$ ) of the metallic melts, including even pure metals, show a decrease in the nearest-neighbor distance to the first atomic coordination shell with increasing temperature. ${ }^{27,28}$ At the same time, the distances in the second, third, and other coordination shells increase with temperature. Such a discrepancy in thermal behavior should be connected with the atomic structure changes in the liquid involving atomic rearrangements in the first and higher-order coordination shells.

Crystallization of different substances has been studied by molecular-dynamics (MD) simulation. ${ }^{29,30}$ At large supercooling, it can be studied directly while at small supercooling, seeding of nucleation is required. ${ }^{31}$ Crystallization of liquid metals has been studied 
by experiment ${ }^{32,33}$ and computer simulation ${ }^{34,35}$ with various potentials. ${ }^{36}$ Among other metals, nickel showed a large supercooling of $341^{37}$ and even $480 \mathrm{~K}^{38}$ using containerless-cooling techniques. Taking into account the melting temperature of nickel (1728 K), the latter value gives a reduced supercooling of 0.28 , which is large for pure metals with a relatively high melting temperature.

Energy barriers for crystallization of pure metals were tested recently, and the nucleation rates were measured and compared with those from computer simulation. ${ }^{39}$ The structure and properties of liquid nickel were also studied extensively by classical MD simulation $^{40,41}$ which allowed to reproduce atomic-scale aspects of crystallization. Evolution of the short-range order and the atomic dynamics of nickel were also studied in the liquid state upon cooling using first-principles MD simulation. ${ }^{42,43}$ The structure was characterized by the coordination number $(\mathrm{CN})$ and bond-angle distributions. The results indicated the existence of so-called distorted icosahedral structures. The structure of supercooled nickel was characterized by complex Frank-Kasper-type polyhedra with coordination numbers of 14-16. ${ }^{42,43}$ The crystallization behavior of other metals was also studied via MD simulation. ${ }^{44}$ In the present manuscript, we deliver a comprehensive research report on the vitrification and crystallization of liquid nickel on cooling, including construction of a time-temperature-transformation (TTT) diagram for the first time.

\section{MODELING PROCEDURE}

A computer simulation using a software package for classical molecular dynamics (LAMMPS) ${ }^{45}$ was used to model the glass transition and crystallization of liquid nickel. Two types of face-centered cubic (fcc) crystalline cells of nickel containing 364500 and 108000 atoms were created and treated in three stages: (a) heating the simulation cell from 300 to $2500 \mathrm{~K}$, (b) holding at $2500 \mathrm{~K}$ for $10 \mathrm{ps}$ (empirically found to be long enough to equilibrate the liquid as the energy and density values stabilized after about 1 ps of holding), and (c) cooling to $300 \mathrm{~K}$ at a cooling rate of about $5 \times 10^{14}$, $5 \times 10^{13}, 5 \times 10^{12}$, or $5 \times 10^{11} \mathrm{~K} \mathrm{~s}^{-1}$. Melting is confirmed by the changes in the radial distribution function (RDF) and rapid stabilization of the density and energy as a function of time. The cells of typically 108000 atoms (in some cases, the cells of other sizes studied for comparison) were also heated to $2500 \mathrm{~K}$, held for 10 , 20 , or 30 ps (for better statistics to avoid the effect of thermal history), and cooled at $5 \times 10^{13} \mathrm{~K} \mathrm{~s}^{-1}$ to a certain temperature to study isothermal crystallization. An embedded-atom method potential ${ }^{46}$ was used in the simulation, which was carried out with 1 fs time steps under periodic boundary conditions with temperature and pressure stabilized using a thermostat ${ }^{47,48}$ and a barostat. ${ }^{49}$ The temperature during simulation was maintained at $\pm 5 \mathrm{~K}$, while the pressure oscillated from -100 to $100 \mathrm{MPa}$. The system did not evaporate in the molten state (as should have happened at zero or negative pressure) owing to the rapid oscillation from negative to positive pressure within a femtosecond time scale, which did not allow a gaseous phase to nucleate. To prove that there is no significant pressure effect on crystallization, an excessive $100 \mathrm{MPa}$ pressure was also applied and no variation in the crystallization kinetics was detected. Much higher pressure values were found to be required to influence the crystallization of metallic glasses ${ }^{50}$ and liquids. The "OVITO" software package was used to visualize and analyze the simulation results.
An adaptive Common-Neighbor Analysis (CNA), ${ }^{52,53}$ in which a characteristic cage signature is computed from the topology of bonds that connect the surrounding neighbor atoms within a specified cutoff distance, was used to analyze structural changes within the simulation cells. For densely packed structures such as fcc, the cutoff distance is set to be halfway between the first- and second-neighbor coordination shells. Three characteristic numbers are computed for each of the $N$ first-neighbor bonds of the central atom: the number of neighboring atoms the central atom and its bonded neighbor have in common; the total number of bonds between these common neighbors; and the number of bonds in the longest chain of bonds connecting the common neighbors. These values are compared with a set of reference signatures to assign a structural type to the central atom.

\section{RESULTS}

The potential was tested by simulation of 108000 atoms inside a cube-shaped cell. Half of the atoms belonged to an fcc crystal relaxed at $300 \mathrm{~K}$ and the other half to a liquid phase relaxed for $100 \mathrm{ps}$ at $3000 \mathrm{~K}$ with the interface in the middle. By movement of the interface on subsequent annealing for 100 ps at different temperatures, the melting/liquidus temperature was found to be slightly above $1725 \mathrm{~K}$ which is consistent with the experimental value for nickel.

Then, two fcc-type lattice cells containing 364500 and 108000 atoms were processed as stated above and cooled to $300 \mathrm{~K}$ at a cooling rate of $\sim 5 \times 10^{13}$ and $\sim 5 \times 10^{12} \mathrm{~K} \mathrm{~s}^{-1}$, respectively. The atoms participating in fcc-type ordered clusters found according to the CNA method are shown in Fig. 1. The number of ordered clusters in the liquid state at $2500 \mathrm{~K}$ was negligibly small. In addition, a cell containing 108000 atoms was also cooled at $\sim 5 \times 10^{13} \mathrm{~K} \mathrm{~s}^{-1}$ and the result was similar to that for the cell containing 364500 atoms. The cells cooled at $\sim 5 \times 10^{13} \mathrm{~K} \mathrm{~s}^{-1}$ had a glassy structure. Those cooled at $\sim 5 \times 10^{12} \mathrm{~K} \mathrm{~s}^{-1}$ showed the formation of a large number of fcc-type nuclei, while those cooled at $\sim 5 \times 10^{11} \mathrm{~K} \mathrm{~s}^{-1}$ crystallized completely in the temperature region from 1000 to $800 \mathrm{~K}$. About 850 atoms having fcc order found for the cell in Fig. 1(a) at $300 \mathrm{~K}$ (cooled at $\sim 5 \times 10^{13} \mathrm{~K} \mathrm{~s}^{-1}$ ) are about $0.2 \%$ of the total number, and they are well separated in space compared to those in Fig. 1(b) obtained at $\sim 5 \times 10^{12} \mathrm{~K} \mathrm{~s}^{-1}$ where fcc-type ordered nuclei/nanoparticles are already seen.

Figure 2(a) shows a series of the reduced radial distribution or pair distribution functions $[\mathrm{PDFs}(R)]$ obtained at different temperatures using a cell containing 364500 atoms cooled at $\sim 5 \times 10^{13} \mathrm{~K} \mathrm{~s}^{-1}$, including one derived for the initial crystalline fcc cell obtained at $540 \mathrm{~K}$ on heating before melting. The $\operatorname{PDF}(R)$ is obtained from the radial distribution function, $\operatorname{RDF}(R)$, as

$$
\operatorname{PDF}(R)=\operatorname{RDF}(R) / 4 \pi R^{2} \rho_{0},
$$

where $R$ is the distance from an arbitrary atom and $\left(\rho_{0}\right)$ is an average atomic number density per unit volume and represents the radial variation of the atomic number density $\rho(R)$ normalized by $4 \pi R^{2} \rho_{0}$. Although some peaks for crystalline nickel correspond well to those of the liquid/glassy phases, the crystalline peaks at $0.35,0.56$, 0.75 , and $0.98 \mathrm{~nm}$ correspond to the minima of $\operatorname{PDFs}(R)$ for the disordered phases. 


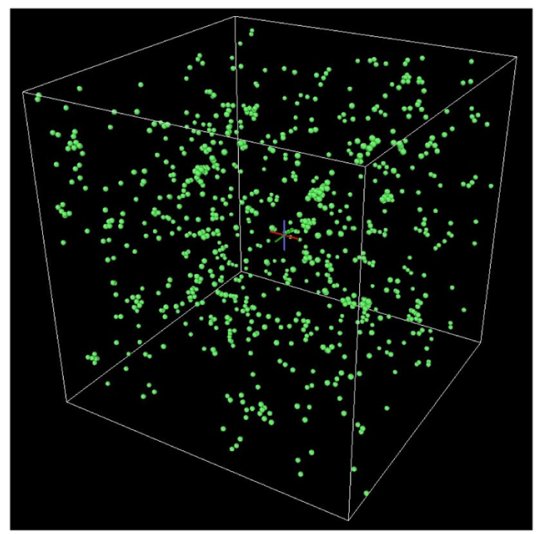

(a)

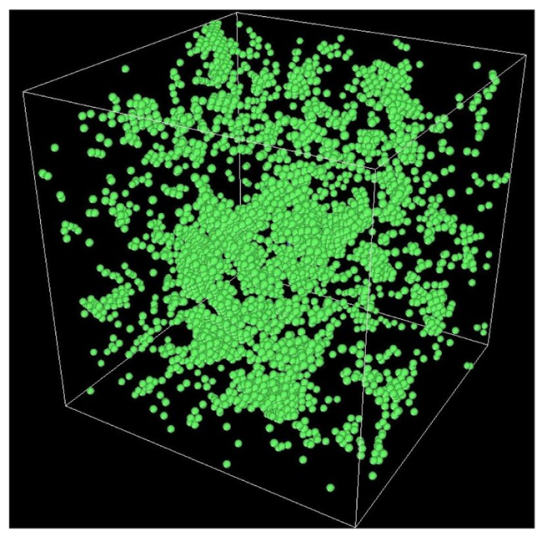

(b)
FIG. 1. Cells (a) of cube edge length $16.1 \mathrm{~nm}$ containing 364500 atoms and (b) of edge length $10.7 \mathrm{~nm}$ containing 108000 atoms, at $300 \mathrm{~K}$, cooled at $5 \times 10^{13}$ and $5 \times 10^{12} \mathrm{~K} \mathrm{~s}^{-1}$, respectively. Green spots indicate the atomic positions with nearly fcc order according to the CNA. While only the beginning of clustering can be seen in (a), fcc nickel nanoparticles are already formed in (b) although the radial distribution function created from this simulation cell still corresponds to a glassy state. The volume fraction of the atoms involved in the nanoparticles in (b) is $3.7 \%$.
Fitting of the initial four $\operatorname{PDF}(R)$ maxima from 0.2 to $0.95 \mathrm{~nm}$ (the baseline was corrected to give zero at each end of the $x$-axis as shown) using six Gaussian functions (including two for the first maximum and two for the second one) produced a reasonable

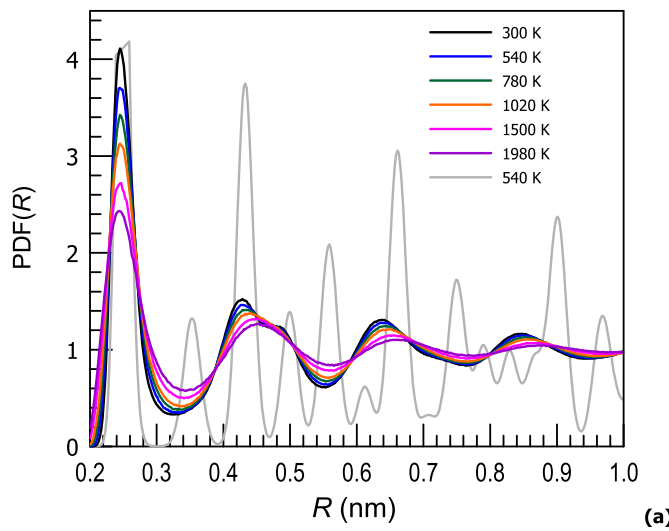

(a)

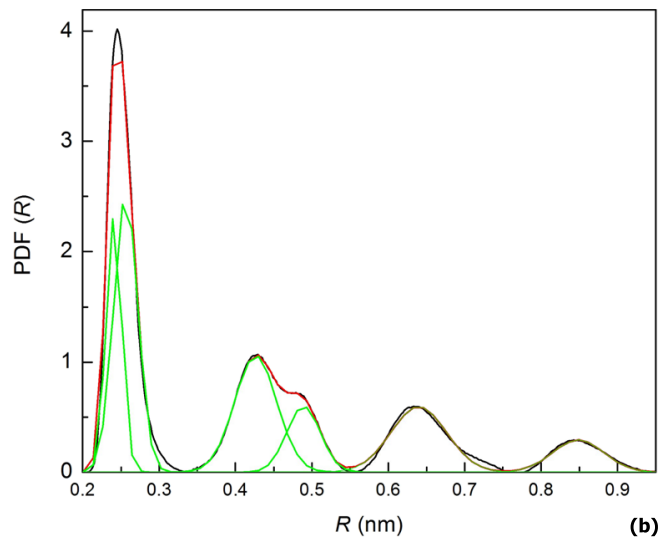

FIG. 2. (a) A series of $\operatorname{PDF}(R)$ functions obtained at different temperatures using a cell containing 364500 atoms cooled at $5 \times 10^{13} \mathrm{~K} \mathrm{~s}^{-1}$. The $\operatorname{PDF}(R)$ of a crystalline phase (gray line) is obtained on initial heating at $540 \mathrm{~K}$. (b) Fitting of the $\operatorname{PDF}(R)$ (black) obtained for a cell at $300 \mathrm{~K}$ with six Gaussian peaks (green). The resulting fitting profile is marked in red. correspondence with the original $\operatorname{PDF}(R)$ plot [Fig. 2(b)] and was used to monitor the thermal expansion/contraction of the cell. The position of the first maximum corresponds very well to that of nickel with the Goldschmidt atomic radius of $r_{\mathrm{Ni}}=0.125 \mathrm{~nm}^{54}$ and an interatomic distance of $0.250 \mathrm{~nm}$.

The thermal contraction on cooling was monitored using the shift in the PDF peaks (Fig. 3). The left shoulder (the first Gaussian peak) of the first maximum $\left(\mathrm{P}_{1}\right)$ shifts to a higher value on cooling until the glass-transition temperature $\left(T_{\mathrm{g}}\right)$ is reached. The right shoulder (the second Gaussian peak) of the first maximum $\left(\mathrm{P}_{2}\right)$ position is nearly unchanging. The second maximum separated into two other Gaussians as $\mathrm{P} 21_{1}$ and $\mathrm{P} 22_{2}$ shows a different behavior of $\mathrm{P} 2_{1}$, while the $\mathrm{P} 2_{2}$ peak position is nearly constant. The centers of mass ( $R$ values) of P3 and P4 (5th and 6th Gaussian peaks) increase with temperature. Such changes illustrate variation of the atomic structure in the liquid state.

The linear coefficient of thermal expansion $\left(\alpha_{\mathrm{p}}\right)$ estimated by the shift of the position $\left(R_{i}\right)$ of $\mathrm{P} 3$ and $\mathrm{P} 4$ in the liquid state using the following equation:

$$
\alpha_{\mathrm{p}}=\left(1 / R_{i}\right)\left(\mathrm{d} R_{i} / \mathrm{d} T\right),
$$

is about $2 \times 10^{-5} \mathrm{~K}^{-1}$. From the variation of the simulation cell size $(L)$ with temperature, the linear thermal expansion coefficient $\alpha_{\mathrm{c}}=(1 / L)(\mathrm{d} L / \mathrm{d} T)$ is found to be $1.6 \times 10^{-5} \mathrm{~K}^{-1}$ in the glassy state (which corresponds quite well to that of crystalline nickel $1.34 \times 10^{-5} \mathrm{~K}^{-1}$ ) 55 and $2.4 \times 10^{-5} \mathrm{~K}^{-1}$ in the liquid state (which is underestimated compared to that of liquid nickel which is $\left.5.6 \times 10^{-5} \mathrm{~K}^{-1}\right) .56$

An empirical criterion for the glass transition based on $\operatorname{PDF}(R)$ is described by

$$
\mathfrak{R}=\operatorname{PDF}(R)_{\min } / \operatorname{PDF}(R)_{\max },
$$

where $\operatorname{PDF}(R)_{\max }$ and $\operatorname{PDF}(R)_{\min }$ indicate the first maximum and first minimum of $\operatorname{PDF}(R)$, respectively. ${ }^{58}$ The plot of $\Re$ vs temperature is shown in Fig. 4(a). The intersection point of two fitting lines produced by segmented fitting using a computer program R: an environment for data analysis and graphics ${ }^{59}$ and Akaike criterion ${ }^{60}$ for segmented regression, ${ }^{61}$ indicates that $T_{\mathrm{g}}$ is $\sim 930 \mathrm{~K}$.

$\operatorname{RDFs}(R)$ were also produced using the atomic number density values and the atomic positions in the simulated cells. The resulting 

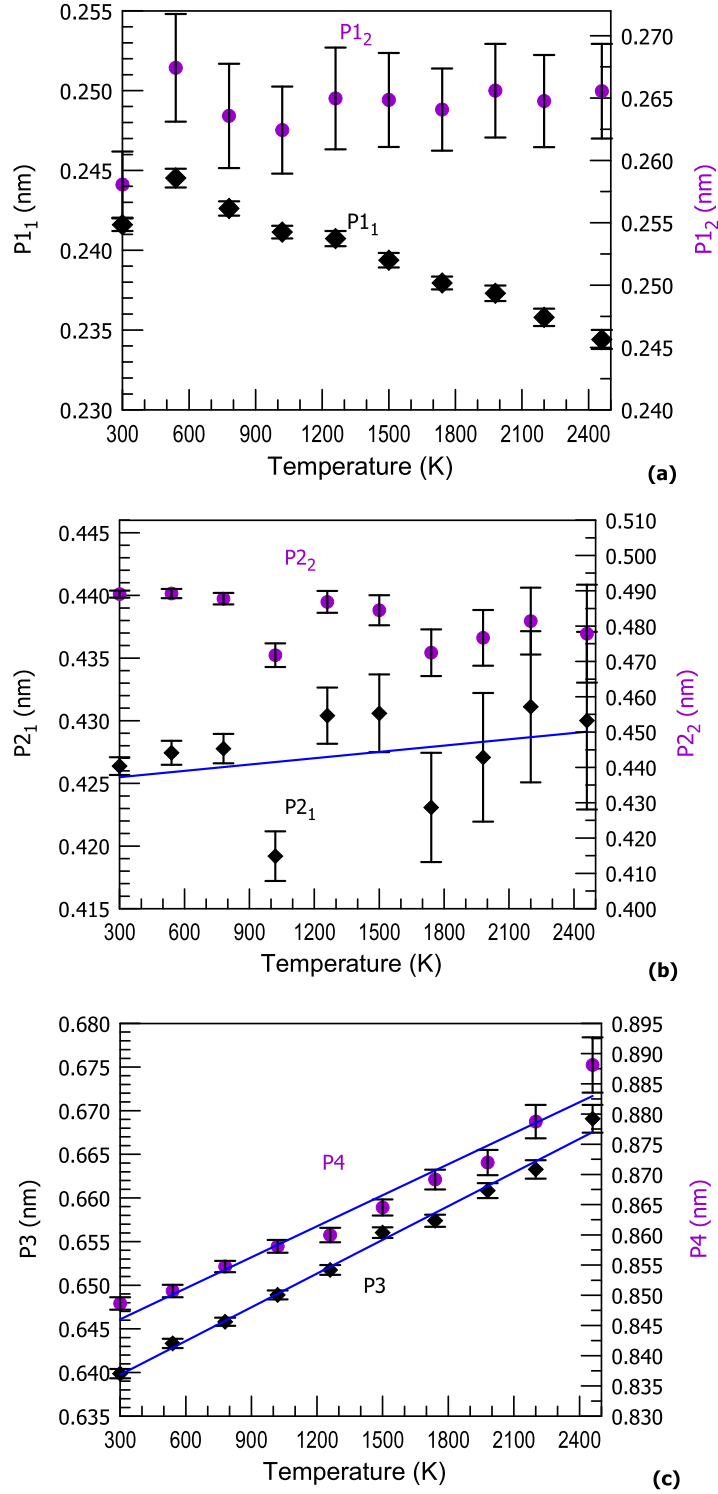

FIG. 3. $\operatorname{PDF}(R)$ peaks shift in the first $\left(P 1_{1,2}\right)(a)$, second $\left(P 2_{1,2}\right)(b)$, as well as third (P3) and fourth (P4) (c) coordination shells as a function of temperature calculated from the PDFs; see the one at $300 \mathrm{~K}$ shown in Fig. 2.

coordination number $(\mathrm{CN})$ - the number of atoms in the first coordination shell in the glassy state calculated by integration of $\operatorname{RDF}(R)$ values up to the first minimum of $\operatorname{RDF}(R)$-was estimated to be 12.9. This is higher than 12 because it is partly affected by the atoms which in a crystal would be located in the second coordination shell. The $\mathrm{CN}$ decreases with increasing temperature in accordance with earlier computational ${ }^{62}$ and experimental works ${ }^{63,64}$ and was found to be 12 at the equilibrium liquidus temperature.

The number of atoms $(N)$ that are the centers of fcc-type clusters according to the CNA is shown as a function of temperature on continuous cooling in Fig. 4(b). The temperature dependence is
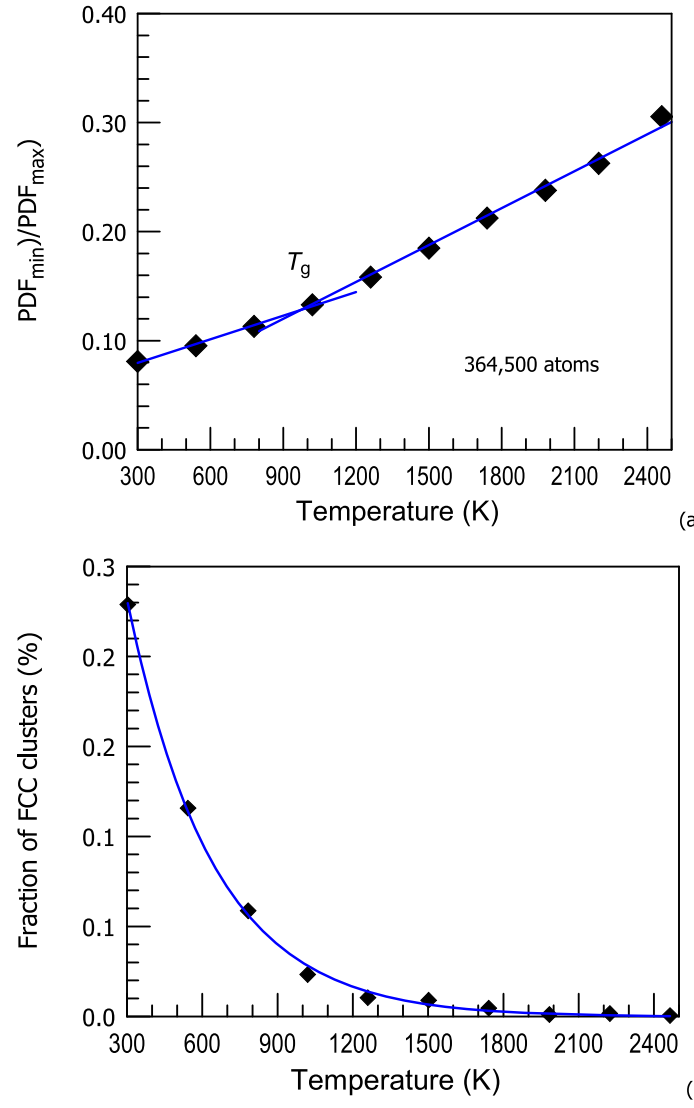

(b)

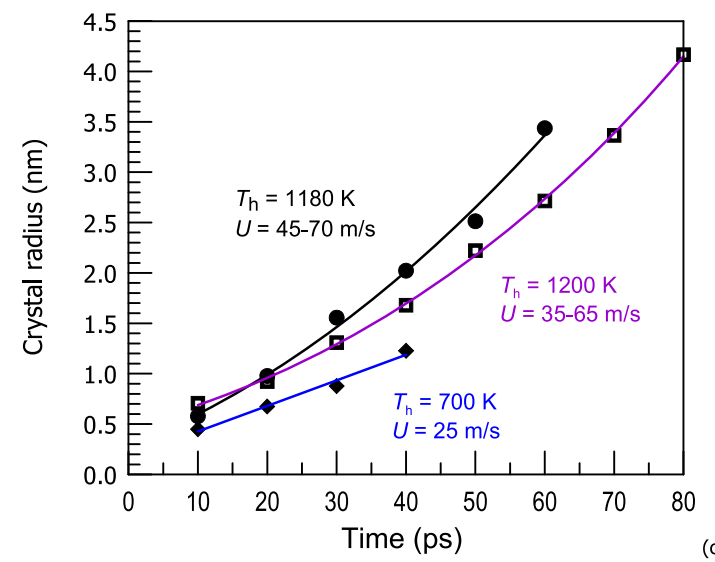

FIG. 4. $\mathfrak{R}=\operatorname{PDF}(R)_{\min } / P D F(R)_{\max }$ as a function of temperature (a). The fraction of atoms with an fcc local environment according to the CNA (b) on cooling, as shown in Fig. 1(a) for $300 \mathrm{~K}$. The data are obtained for a cell containing 364500 atoms cooled at $5 \times 10^{13} \mathrm{~K} \mathrm{~s}^{-1}$. The size of the single nickel crystals as a function of time at $700 \mathrm{~K}, 1180$, and $1200 \mathrm{~K}(\mathrm{c}) . T_{\mathrm{h}}$ is the holding/annealing temperature.

fitted quite well with the exponential decay function,

$$
N=N_{0}+A \cdot \exp (-T / B),
$$

by using the fitting parameters $N_{0}, A$, and $B$ with respective values of $-0.8 \pm 4.9,2029 \pm 60$, and $342 \pm 10$. In a liquid above its 
melting temperature, the cluster populations are expected to be in equilibrium and independent of time. In contrast, below the melting temperature, there is no equilibrium population, and the number that is measured is a function of time and dependent on a number of kinetic factors. Thus, one would not expect a single exponent fitting: the data above the melting temperature should be independent of the cooling rate, and the data below the melting temperature should be dependent on the cooling rate. However, in the present case, the sample visibly vitrified, i.e., there were no postcritical nuclei at room temperature at this particular cooling rate, and the clusters represent fcc coordinated atoms which are spatially separated without clustering [see Fig. 1(a)].

The phase transformation (crystallization) kinetics is shown in Figs. 5(a)-5(d). The cells having 108000 atoms were melted, cooled at $\sim 5 \times 10^{13} \mathrm{~K} \mathrm{~s}^{-1}$, and kept at constant temperatures until crystallization took place. The energy of the system changes in the opposite way to the density and both can be used to monitor the phase transformation [Fig. 5(a)]. At high temperatures, the results of individual computational runs differ significantly [Fig. 5(a)], while in the middle and low-temperature range, the curves of independent MD simulation runs almost superpose on each other [Figs. 5(b)5(d)]. The density (and volume) changes in the cells containing 364000 and 864000 atoms at $1100 \mathrm{~K}$ take place more slowly, likely owing to a larger cell volume to be filled with the crystallizing volume.

The growing nickel crystals have a nearly spherical "potato" type shape [see the inset in Fig. 5(a)]. The growth rate $(U)$ of a growing single crystal determined using an approximate crystal radius $\left(R_{\mathrm{a}}\right)$ as a function of time $(t)\left(U=\mathrm{d} R_{\mathrm{a}} / \mathrm{d} t\right)$ is found to be not constant, in general, and slightly increases with radius, at least at high temperature [Fig. 4(c)]. This arises from the Gibbs-Thomson effect as noted in a study of non-steady state/transient nucleation. ${ }^{6}$ It is possible that the growth rate is also not constant at lower temperatures, but the growing crystals impinge quickly owing to a high nucleation rate. The maximum $U$ values are found to be about $65,70,60,45,45,35,25$, and $20 \mathrm{~m} \mathrm{~s}^{-1}$ at 1200, 1180, 1100, $1000,900,800,700$, and $500 \mathrm{~K}$, respectively. The values of $U$ are slightly lower than the values observed before. ${ }^{66,67}$ Also, the crystal growth rate was scattered from crystal to crystal, likely owing to varying local order in the surrounding environment and the lattice defects (hcp stacking faults) within the growing crystals. Another reason is the impingement of growing crystals with other crystals or nuclei of different crystallographic orientations as was suggested recently.

In the case of pure metals, the interface-controlled growth rate can either be diffusion- or collision-limited. ${ }^{33,65}$ For example, the diffusion coefficient $(D)$ measured using mean-square atomic displacements at $1100 \mathrm{~K}$ is $\sim 5 \times 10^{-10} \mathrm{~m}^{2} \mathrm{~s}^{-1}$. According to formula $U=D / \lambda$, where $\lambda$ is the jump distance or atomic diameter of $0.25 \mathrm{~nm},{ }^{33}$ the diffusion-limited growth rate should be $\sim 2 \mathrm{~m} \mathrm{~s}^{-1}$. Taking into account the abovementioned measured $U$ value of $60 \mathrm{~m} \mathrm{~s}^{-1}$, one can see that it is not diffusion-limited (although still much smaller than the collision-limited value of order $\left.10^{3}-10^{4} \mathrm{~m} \mathrm{~s}^{-1}\right) .^{33}$ Also, diffusion is quite fast even at $300 \mathrm{~K}$, $D=8 \times 10^{-13} \mathrm{~m}^{2} / \mathrm{s}$, this high value possibly arising because the atomic structure has not been fully relaxed.

The Kolmogorov-Johnson-Mehl-Avrami plot ${ }^{69,70}$ of $\ln (\ln (1 /(1-x)))$ as a function of $\ln (t)$, where $x$ is the fraction of the
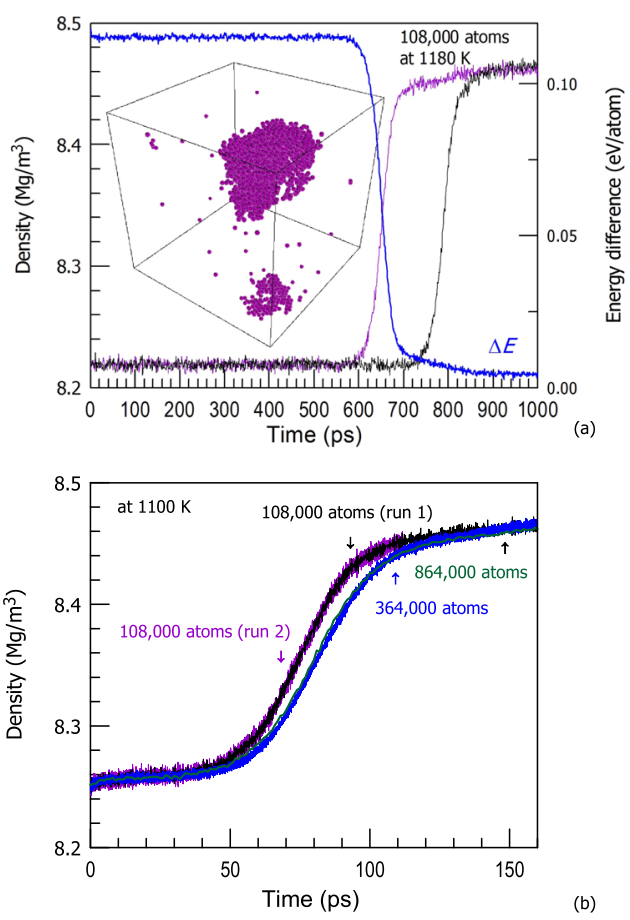

(b)
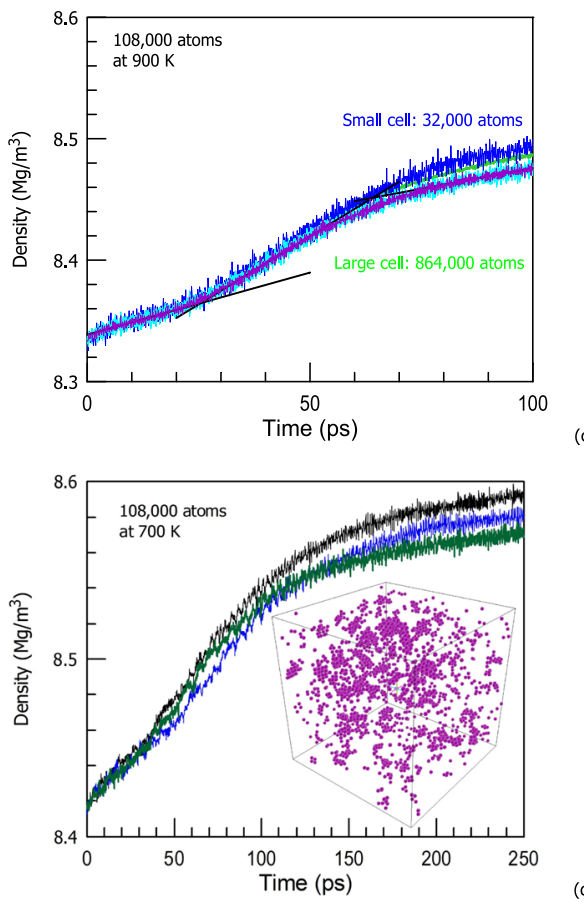

(c)

(d)

FIG. 5. Density change as a function of time for pure nickel at 1180 (a), $1100 \mathrm{~K}$ (b), $900 \mathrm{~K}$ (c), and $700 \mathrm{~K}$ (d) for the cell containing 108000 atoms. Lines of different colors indicate the results of 2 independent computational runs. Other cells sizes were also used for comparison. The inset in (a) shows a crystalline particle growing during phase transformation. (a) The blue line also indicates the energy difference $(\Delta E)$ for the purple curve, shown for example, as marked. The inset in (d) represents the fcc type atomic environment after $30 \mathrm{ps}$ of waiting time at $700 \mathrm{~K}$ indicating several nuclei/particles. 
crystalline phase and $t$ is the time after completion of the incubation period, is shown for $1180 \mathrm{~K}$ in Fig. 6(a) and yields the exponent $(n)^{69}$ of 4.2 . This value indicates steady-state nucleation and 3-dimensional interface-controlled growth of crystals in accordance with the theory for polymorphic transformation (i.e., without changes in chemical composition). ${ }^{69}$ However, at $1100 \mathrm{~K}, n=3$, while at $900 \mathrm{~K}$ [Fig. 5(c)], $n$ is only 2.2. At $1100 \mathrm{~K}$ and below, glassy structure relaxation still takes place when crystallization starts. Such low values of $n$ indicate the possible presence of pre-existing nuclei in these temperature ranges (formed on cooling to the holding temperature), which is consistent with the plot shown in Fig. 6(b) indicating formation of fcc clusters as a function of time at $700 \mathrm{~K}$.

From the isothermal crystallization plots, the beginning time $\left(t_{\mathrm{b}}\right)$, also called an incubation time, and finish $\left(t_{\mathrm{f}}\right)$ time of
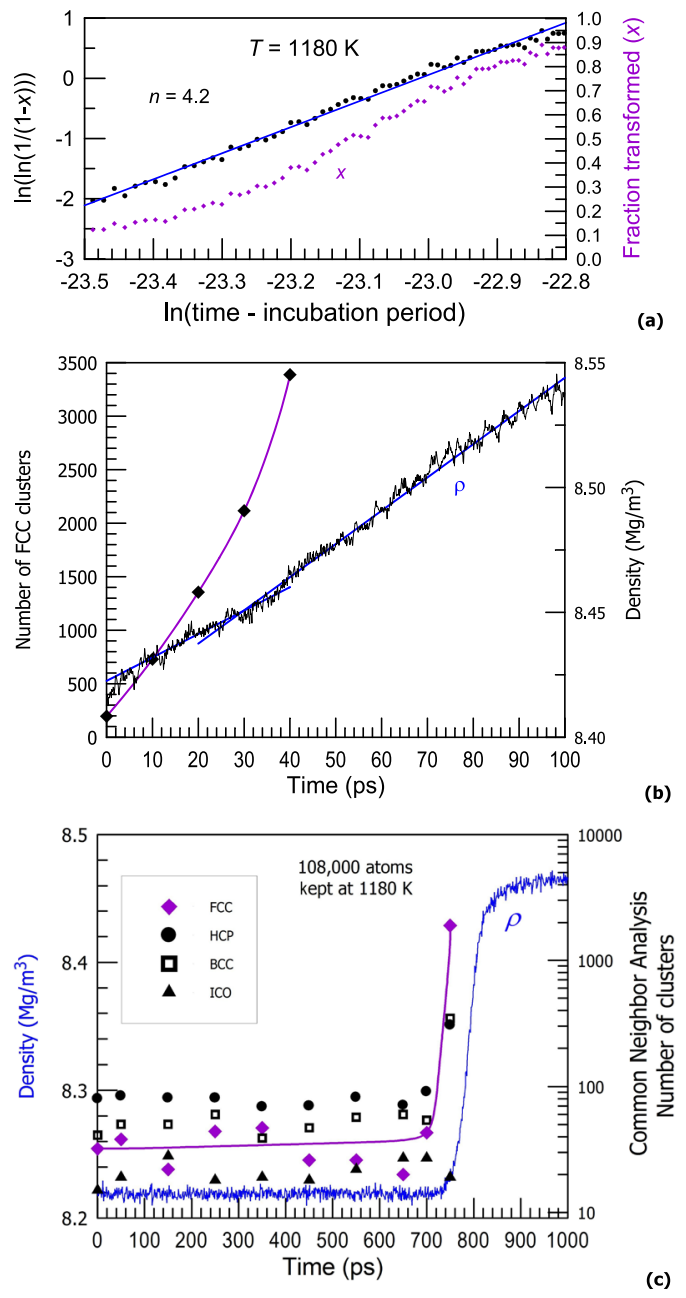

FIG. 6. (a) The Avrami plot and the fraction transformed at $1180 \mathrm{~K}$. (b) The number of fcc clusters according to the CNA as a function of time at $700 \mathrm{~K}$ (purple line) and density variation (black line). The blue lines are the tangents used to determine the point of change in the slope. (c) The number of fcc, hcp, bcc, and ICO (icosahedral) clusters according to the CNA as a function of time at $1180 \mathrm{~K}$. No variation of cluster population is seen until the first nucleus is formed. transformation were calculated using two corresponding tangents [see Figs. 5(c) and 6(b), for example] to the plot before and after the inflection point. Note that actual transformation time is longer because the two tails of the S-curve are not taken into consideration when two tangents are applied. The results of several different computational runs are also shown for comparison. However, it is not easy to detect the incubation period for each plot below $800 \mathrm{~K}$ owing to the long relaxation time manifested in constant variation of the baseline [see Fig. 5(d), for explanation]. Above $1200 \mathrm{~K}$, it was not possible to observe crystallization until after $3 \mathrm{~ns}$ of computations.

A time-temperature-transformation (TTT) diagram plotted in Fig. 7 indicates that crystallization of nickel takes place very fast over a wide temperature range down to room temperature. Also, owing to the high-enough nucleation rates, the shape of the crystallization curves [Figs. 5(b)-5(d)], the incubation time, and reaction time below $1100 \mathrm{~K}$ are well reproduced by the independent MD runs. However, at a higher temperature, the incubation time obtained for different runs is scattered [Figs. 5(a) and 7] owing to a low number density of nuclei compared to the cell size. Below $800 \mathrm{~K}$, it is not so easy to detect the corresponding values of $t_{\mathrm{b}}$ due to significant relaxation [see Fig. 5(d), for example] in the supercooled liquid/glass and these values should be treated with care. Also, the results of the CNA indicate that there are no fcc-type clusters in the liquid phase at high temperature on the initial melting while, for example, 196 scattered fcc-type clusters (only nearest coordination, not groups of atoms) already exist after cooling to $700 \mathrm{~K}$ [the curves are shown in Fig. 5(d)]. However, then the number of fcc clusters increases to $729,1355,2116$, and 3388 after 10, 20,30, and 40 ps of holding at $700 \mathrm{~K}$ [Fig. 6(b)]. Thus, postcritical nuclei can already be clearly observed after 30 ps of waiting time [Fig. 5(d) (inset)]. This result indicates that the energy barrier for nucleation may be absent at low temperature when only slow kinetics restricts crystallization. On the other hand, at a high-enough temperature of $1180 \mathrm{~K}$ [Fig. 6(c)], when the energy barrier is high enough, the number of crystal-like atomic zones remains constant within the incubation period until the first nucleus is formed at $\sim 750 \mathrm{ps}$. The TTT diagram allows us to calculate the approximate critical cooling rate

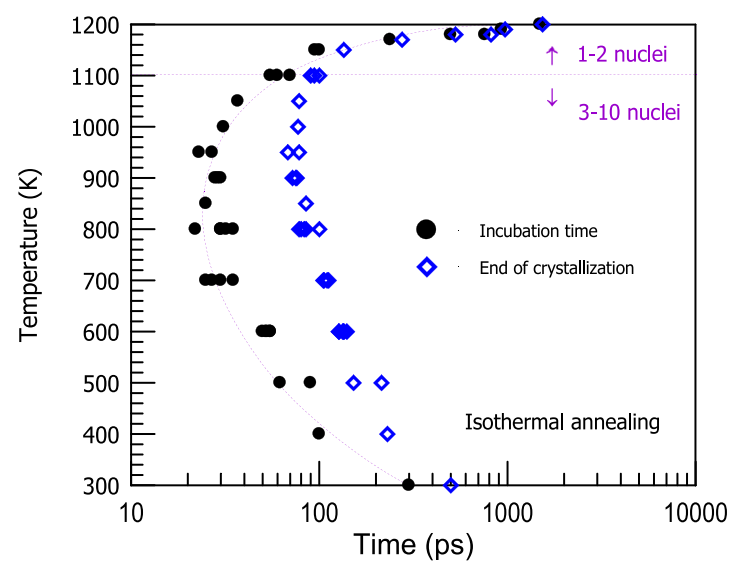

FIG. 7. Time-temperature-transformation (TTT) diagram for supercooled liquid nickel. The cells contained 108000 atoms. 
using the minimum incubation time $\left(t_{\mathrm{im}}\right)$ of $20 \mathrm{ps}$ and the liquidus temperature $\left(T_{1}\right)$ of $1728 \mathrm{~K}$. The resulting value $\left(T_{1}-298\right) / t_{\text {im }}$ of $7.15 \times 10^{13} \mathrm{~K} \mathrm{~s}^{-1}$ is close to the $5 \times 10^{13} \mathrm{~K} \mathrm{~s}^{-1}$ obtained on continuous cooling.

\section{DISCUSSION}

Amorphization of an atomic cell of pure nickel upon MD simulation was attained at cooling rates exceeding $5 \times 10^{12} \mathrm{~K} \mathrm{~s}^{-1}$. The glass-transition temperature of $930 \mathrm{~K}$ estimated by the change in the slope of $\Re$ as a function of temperature is slightly lower than the equivolume/isochoric $T_{\mathrm{g}}$ determined before ${ }^{71}$ and confirmed by classical ${ }^{72}$ and $a b$ initio $^{73} \mathrm{MD}$ simulation. By analogy with Kauzmann's entropy crisis, ${ }^{74}$ a liquid metal should not have a higher density, at a given temperature, than its crystalline counterpart. The fcc structure is one of the most densely packed, and thus, the monoatomic liquid phase should have vitrified above the isochoric (equivolume) $T_{\mathrm{g}}$. A slightly lower value of equivolume/isochoric $T_{\mathrm{g}}$ can be explained by the fact that although the volume changes, $\mathrm{d} V / \mathrm{d} T$, of the crystalline phase are very well reproduced by simulation, the volumetric and linear thermal expansion coefficients for the liquid phase in the present simulation are smaller than the experimental values. On the other hand, according to the density variation plot at constant temperature, the relaxation time (time related to stabilization of a certain property of a glass: energy, density, etc.) at $1100 \mathrm{~K}$ becomes longer than the observation time (owing to low atomic mobility) [Figs. 5(b) and 5(c)] which also corresponds quite well to the observed $T_{\mathrm{g}}$.

In earlier work, the nucleation of crystals in liquid nickel (a cell containing 12000 atoms) on continuous cooling also took place at a cooling rate below $5 \times 10^{12} \mathrm{~K} \mathrm{~s}^{-1}$. ${ }^{75}$ Similar critical cooling rate values were obtained in other work on crystallization of pure metals by MD simulation. ${ }^{76,77}$ However, experimental results suggest a significantly lower critical cooling rate for glass formation in nickel and some other pure metals. ${ }^{78,79}$ The critical cooling rate values obtained from MD simulation also exceed those predicted by thermodynamic calculations. ${ }^{80}$ However, since these calculations underestimate $T_{\mathrm{g}}$ of nickel (the suggested values are $732 \mathrm{~K}^{78}$ and $509 \mathrm{~K}^{80}$ ), their validity should likely be reconsidered.

Below $1100 \mathrm{~K}$, owing to high-enough nucleation rate, the incubation time and reaction time in the TTT diagram are quite well reproduced by independent MD runs. On the other hand, it becomes difficult to detect homogeneous nucleation above about $0.7 T_{1}$. Also, below $800 \mathrm{~K}$, it is difficult to distinguish the increase in density owing to the beginning of crystallization from that related to structural relaxation in the glassy phase. It was suggested in Ref. 75 that nonclassical nucleation takes place in liquid nickel. This finding is consistent with the low Avrami exponent ( $n$ ) found in the present research below $1100 \mathrm{~K}$, while at a higher temperature, it rather follows the classical form with $n=4$.

In general, nanocrystallization is expected at large supercooling, especially when the growth is diffusion-controlled. ${ }^{81,82}$ However, crystallization of pure metals is by definition a polymorphic phase transformation, which should lead to rather large crystals owing to high $U$ values ${ }^{83}$ unless very large supercooling is achieved, as in the case of MD simulation. In the present work, the number of nuclei formed within the MD nickel cells of about $10 \times 10$ $\times 10 \mathrm{~nm}^{3}$ (108000 atoms) ranges from 1 to 10 , leading to a number density of precipitates of the order of $10^{24} \mathrm{~m}^{-3}$. The high number density of nuclei observed leads to good reproducibility of the crystallization traces at $T_{\mathrm{g}}$ and below. Such high number densities of precipitates are more or less typical for the crystallization of pure metals at extremely large supercooling $\left(\Delta T_{\mathrm{u}}\right)$ of about $0.55 T_{1}$ and at even lower temperatures, which are unattainable experimentally for liquids, but quite common in some metallic glasses which crystallize in a similar reduced temperature range. ${ }^{84}$

\section{CONCLUSIONS}

Vitrification and crystallization processes in liquid nickel were studied in detail using MD simulation. The glass transition was monitored using the radial distribution functions and cluster analysis. A decrease in the interatomic distances takes place within the second (left shoulder) of third and fourth coordination shells on cooling, while expansion is observed within the first coordination shell in the liquid state. Such changes highlight the variation of the atomic structure in the liquid state. The coordination number increases on cooling, from 12 above the liquidus to 12.9 at $300 \mathrm{~K}$.

The crystallization kinetics was analyzed under isothermal conditions by monitoring density and energy variation as a function of time. The growing nanocrystals have an irregular but spheroidal "potato" shape. The growth rate of the nanocrystals is nearly constant, but at $1200 \mathrm{~K}$, it slightly increases with radius, in agreement with theoretical predictions. The crystal growth rate was scattered from crystal to crystal likely owing to varying local order in the surrounding environment and the lattice defects (hcp stacking faults) within the growing crystals.

The temperature $T_{\mathrm{N}}$ corresponding to the minimum incubation period in the TTT diagram is 700-900 K. However, it is difficult to determine clearly the incubation time below $900 \mathrm{~K}$ owing to structural relaxation in the glass at low temperature. Also, homogeneous nucleation becomes difficult to detect above about $0.7 T_{1}$ within reasonable simulation time scales (up to $3 \mathrm{~ns}$ ).

The population density of crystalline precipitates forming below $T_{\mathrm{N}}$ is of the order of $10^{24} \mathrm{~m}^{-3}$. Such a high number density of nuclei leads to good reproducibility of the density traces as a function of time.

\section{ACKNOWLEDGMENTS}

The authors gratefully acknowledge the financial support received from the World Premier International Research Center Initiative (WPI), MEXT, Japan; by the Ministry of Education and Science of the Russian Federation in the framework of the "Increase Competitiveness" Program of NUST “MISiS" Grant Nos. K2-2014013 and K2-2017-089); and by the WPI-AIMR Fusion Research Project: "Nucleation-Structural formation dynamics of nanomaterials." D.V.L. also sincerely thanks Alexander Evteev (who suddenly passed away recently) for fruitful discussions and Masayuki Okugawa for his help in the analysis of the validity of the potential and Hiroshi Suito for his valuable help with the statistical analysis of the experimental data. A.L.G. acknowledges financial support through the European Research Council under ERC Advanced Grant "Extend Glass" (Grant No. ERC-2015-AdG-695487). 


\section{REFERENCES}

${ }^{1}$ J. Frenkel, Kinetic Theory of Liquids (Oxford University Press, Oxford, 1946).

${ }^{2}$ P. G. Debenedetti and F. H. Stillinger, "Supercooled liquids and the glass transition," Nature 410, 259-267 (2001).

${ }^{3}$ C. A. Angell, "Structural instability and relaxation in liquid and glassy phases near the fragile liquid limit," J. Non-Cryst. Solids 102, 205-221 (1988).

${ }^{4}$ A. L. Greer, "Metallic glasses. . on the threshold," Mater. Today, 12, 14-22 (2009).

${ }^{\mathbf{5}}$ T. Egami, "Atomistic mechanism of bulk metallic glass formation," J. Non-Cryst. Solids 317, 30-33 (2003).

${ }^{6} \mathrm{D}$. V. Louzguine-Luzgin, "Vitrification and devitrification processes in metallic glasses," J. Alloys Compd. 586, S2-S8 (2014).

${ }^{7}$ M. H. Cohen and G. S. Grest, "Liquid-glass transition, a free-volume approach," Phys. Rev. B 20, 1077-1098 (1979).

${ }^{8}$ D. V. Louzguine-Luzgin and A. Inoue, "The outline of glass transition phenomenon derived from the viewpoint of devitrification process," Phys. Chem. Glasses: Eur. J. Glass Sci. Technol., Part B 50, 27-30 (2009).

${ }^{9} \mathrm{~N}$. Jakse and A. Pasturel, "Local order and dynamic properties of liquid and undercooled $\mathrm{Cu}_{\mathrm{x}} \mathrm{Zr}_{1-\mathrm{x}}$ alloys by ab-initio molecular dynamics," Phys. Rev. B 78, 214204 (2008).

${ }^{10}$ J. E. Velasco, A. Concustell, E. Pineda, and D. Crespo, "Plastic deformation induced anisotropy in metallic glasses: A molecular dynamics study," J. Alloys Compd. 707, 102-107 (2017).

${ }^{11}$ C. Suryanarayana and A. Inoue, Bulk Metallic Glasses (Taylor and Francis Group, LLC, 2011), p. 17.

${ }^{12}$ D. V. Louzguine-Luzgin, "Bulk metallic glasses and glassy/crystalline materials," in Novel Functional Magnetic Materials, Fundamentals and Applications, Volume 231 of the Springer Series in Materials Science, edited by A. Zhukov (Springer International Publishing, Switzerland, 2016), pp. 397-440.

${ }^{13}$ Y. W. Kim, H. M. Lin, and T. F. Kelly, "Solidification structures in submicron spheres of iron-nickel: Experimental observations," Acta Metall. 36, 2525 (1988).

${ }^{14}$ A. V. Evteev, A. T. Kosilov, and E. V. Levchenko, "Atomic mechanisms of pure iron vitrification," J. Exp. Theor. Phys. 99, 522-529 (2004).

${ }^{15} \mathrm{M}$. I. Ojovan, "Ordering and structural changes at the glass-liquid transition," J. Non-Cryst. Solids 382, 79-86 (2013).

${ }^{16} \mathrm{D}$. Cangialosi, "Glass transition and physical aging of confined polymers investigated by calorimetric techniques," in Handbook of Thermal Analysis and Calorimetry (Elsevier, 2018), Vol. 6, pp. 301-337.

${ }^{17} \mathrm{Y}$. Waseda and H. S. Chen, "A structural study of metallic glasses containing boron (Fe-B, Co-B, and Ni-B)," Phys. Status Solidi 49, 387-396 (1978).

${ }^{18}$ D. V. Louzguine-Luzgin, A. R. Yavari, G. Vaughan, and A. Inoue, "Clustered crystalline structures as glassy phase approximants," Intermetallics 17, 477-480 (2009).

${ }^{19}$ A. R. Yavari, N. Nikolov, N. Nishiyama, T. Zhang, A. Inoue, J. L. Uriarte, and G. Heunen, "The glass transition of bulk metallic glasses studied by real-time diffraction in transmission using high-energy synchrotron radiation," Mater. Sci. Eng., A 375-377, 709-712 (2004).

${ }^{20}$ N. A. Mauro, M. L. Johnson, J. C. Bendert, and K. F. Kelton, "Structural evolution in $\mathrm{Ni}-\mathrm{Nb}$ and $\mathrm{Ni}-\mathrm{Nb}-\mathrm{Ta}$ liquids and glasses - A measure of liquid fragility," J. Non-Cryst. Solids 362, 237 (2013).

${ }^{21}$ P. Lamparter, "Structure of metallic glasses," Phys. Scr. T57, 45-63 (1995).

${ }^{22}$ T. Egami and S. J. L. Billinge, Underneath the Bragg Peaks: Structural Analysis of Complex Materials, 1st ed. (Elsevier, Oxford, 2003); 2nd ed. (Elsevier, Oxford, 2012).

${ }^{23}$ A. Hirata, Y. Hirotsu, T. Ohkubo, N. Tanaka, and T. G. Nieh, "Local atomic structure of $\mathrm{Pd}-\mathrm{Ni}-\mathrm{P}$ bulk metallic glass examined by high-resolution electron microscopy and electron diffraction," Intermetallics 14, 903-917 (2006).

${ }^{24}$ D. V. Louzguine-Luzgin, J. Antonowicz, K. Georgarakis, G. Vaughan, A. R. Yavari, and A. Inoue, "Real-space structural studies of $\mathrm{Cu}-\mathrm{Zr}$-Ti glassy alloy," J. Alloys Compd. 466, 106-110 (2008).
${ }^{25}$ D. V. Louzguine-Luzgin, A. R. Yavari, G. Vaughan, and A. Inoue, "Thermal expansion of a glassy alloy studied using a real-space pair distribution function," Appl. Phys. Lett. 88, 121926 (2006).

${ }^{26}$ A. K. Gangopadhyay, M. E. Blodgett, M. L. Johnson, J. McKnight, V. Wessels, A. J. Vogt, N. A. Mauro, J. C. Bendert, R. Soklaski, L. Yang, and K. F. Kelton, "Anomalous thermal contraction of the first coordination shell in metallic alloy liquids," J. Chem. Phys. 140, 044505 (2014).

${ }^{27}$ H. Lou, X. Wang, Q. Cao, D. Zhang, J. Zhang, T. Hu, H.-K. Mao, and J.-Z. Jiang, "Negative expansions of interatomic distances in metallic melts," Proc. Natl. Acad. Sci. U. S. A. 110, 10068 (2013).

${ }^{28}$ D. V. Louzguine-Luzgin, K. Georgarakis, A. Tsarkov, A. Solonin, V. Honkimaki, L. Hennet, and A. R. Yavari, "Structural changes in liquid Fe and Fe-B alloy on cooling," J. Mol. Liq. 209, 233-238 (2015).

${ }^{29} \mathrm{E}$. R. Buckle, "Studies on the freezing of pure liquids. II. The kinetics of homogeneous nucleation in supercooled liquids," Proc. R. Soc. A 261(1305), 189-196 (1961).

${ }^{30}$ J. S. van Duijneveldt and D. Frenkel, "Computer simulation study of free energy barriers in crystal nucleation," J. Chem, Phys. 96, 4655 (1992).

${ }^{31}$ J. R. Espinosa, C. Vega, C. Valeriani, and E. Sanz, "Seeding approach to crystal nucleation," J. Chem. Phys. 144, 034501 (2016).

${ }^{32}$ G. Wilde, J. L. Sebright, and J. H. Perepezko, "Bulk liquid undercooling and nucleation in gold," Acta Mater. 54, 4759-4769 (2006).

${ }^{33}$ K. F. Kelton and A. L. Greer, Nucleation in Condensed Matter: Applications in Materials and Biology (Elsevier, Amsterdam, 2010).

${ }^{34}$ Y. Qi, T. C. Agin, Y. Kimura, and W. A. Goddard III, "Molecular-dynamics simulations of glass formation and crystallization in binary liquid metals: $\mathrm{Cu}-\mathrm{Ag}$ and Cu-Ni," Phys. Rev. B 59, 3527 (1999).

${ }^{35}$ C. Desgranges and J. Delhommelle, "Unusual crystallization behavior close to the glass transition," Phys. Rev. Lett. 120, 115701 (2018).

${ }^{36}$ D. K. Belashchenko, "Computer simulation of liquid metals," Phys.-Usp. 56, 1176-1216 (2013).

${ }^{37}$ R. Willnecker, D. M. Herlach, and B. Feuerbacher, "Containerless undercooling of bulk Fe-Ni melts," Appl. Phys. Lett. 49, 1339-1341 (1986).

${ }^{38}$ D. W. Gomersall, S. Y. Shiraishi, and R. G. Ward, "Undercooling phenomena in liquid metal droplets,” J. Aust. Inst. Metals. 10, 220-224 (1965).

${ }^{39} \mathrm{~J}$. Bokeloh, G. Wilde, R. E. Rozas, R. Benjamin, and J. Horbach, "Nucleation barriers for the liquid-to-crystal transition in simple metals: Experiment vs. simulation," Eur. Phys. J.: Spec. Top. 223, 511-526 (2014).

${ }^{40}$ F. J. Cherne, M. I. Baskes, and P. A. Deymier, "Properties of liquid nickel: A critical comparison of EAM and MEAM calculations," Phys. Rev. B 65, 024209 (2001).

${ }^{41}$ D. K. Belashchenko and O. I. Ostrovskii, "A molecular dynamics study of nickel crystallization at strong supercoolings," Russ. J. Phys. Chem. A 82, 364-375 (2008).

${ }^{42} \mathrm{~N}$. Jakse and A. Pasturel, "Ab initio molecular dynamics simulations of local structure of supercooled Ni," J. Chem. Phys. 120, 6124 (2004).

${ }^{43}$ N. Jakse and A. Pasturel, "Molecular-dynamics study of liquid nickel above and below the melting point," J. Chem. Phys. 123, 244512 (2005).

${ }^{44}$ Y. Ashkenazy and R. S. Averback, "Kinetic stages in the crystallization of deeply undercooled body-centered-cubic and face-centered-cubic metals," Acta Mater. 58, 524-530 (2010).

${ }^{45}$ S. J. Plimpton, "Fast parallel algorithms for short-range molecular dynamics," J. Comput. Phys. 117, 1-19 (1995).

${ }^{46}$ M. I. Mendelev, M. J. Kramer, S. G. Hao, K. M. Ho, and C. Z. Wang, "Development of interatomic potentials appropriate for simulation of liquid and glass properties of $\mathrm{NiZr}_{2}$ alloy," Philos. Mag. 92, 4454-4469 (2012).

${ }^{47}$ S. Nosé, "A unified formulation of the constant temperature molecular dynamics methods," J. Chem. Phys. 81, 511-519 (1984).

${ }^{48}$ W. G. Hoover, "Canonical dynamics: Equilibrium phase-space distributions," Phys. Rev. A 31, 1695-1697 (1985).

${ }^{49}$ H. J. C. Berendsen, J. P. M. Postma, W. F. van Gunsteren, A. DiNola, and J. R. Haak, "Molecular dynamics with coupling to an external bath," J. Chem. Phys. 81, 3684-3690 (1984). 
${ }^{50}$ J. Z. Jiang, J. S. Olsen, L. Gerward, S. Abdali, J. Eckert, N. Schlorke-de Boer, L. Schultz, J. Truckenbrodt, and P. X. Shi, "Pressure effect on crystallization of metallic glass $\mathrm{Fe}_{72} \mathrm{P}_{11} \mathrm{C}_{6} \mathrm{Al}_{5} \mathrm{~B}_{4} \mathrm{Ga}_{2}$ alloy with wide supercooled liquid region," J. Appl. Phys. 87, 2664 (2000).

${ }^{51}$ A. Stukowski, "Visualization and analysis of atomistic simulation data with OVITO-The open visualization tool," Modell. Simul. Mater. Sci. Eng. 18, 015012 (2010).

${ }^{52} \mathrm{~J}$. D. Honeycutt and H. C. Andersen, "Molecular dynamics study of melting and freezing of small Lennard-Jones clusters," J. Phys. Chem. 91, 4950-4963 (1987).

${ }^{53}$ A. Stukowski, "Structure identification methods for atomistic simulations of crystalline materials," Modell. Simul. Mater. Sci. Eng. 20, 045021 (2012).

${ }^{54}$ O. N. Senkov and D. B. Miracle, "Effect of the atomic size distribution on glass forming ability of amorphous metallic alloys," Mater. Res. Bull. 36, 2183 (2001).

${ }^{55}$ Smithells Metals Reference Book, 8th ed., edited by W. F. Gale and T. C. Totemeier (Elsevier Butterworth-Heinemann Ltd., Oxford, UK, 2004), p. 14-2.

${ }^{56}$ Smithells Metals Reference Book, 8th ed., edited by W. F. Gale and T. C. Totemeier (Elsevier Butterworth-Heinemann Ltd., Oxford UK, 2004), p. 14-10.

${ }^{57} \mathrm{~J}$. Brillo and I. Egry, "Density determination of liquid copper, nickel, and their alloys," Int. J. Thermophys. 24, 1155 (2003).

${ }^{58} \mathrm{H}$. H. Wendt and F. F. Abraham, "Empirical criterion for the glass transition region based on Monte Carlo simulations," Phys. Rev. Lett. 41, 1244-1246 (1978). ${ }^{59}$ See https://www.r-project.org for downloading the software/program.

${ }^{60} \mathrm{H}$. Akaike, "A new look at the statistical model identification," IEEE Trans. Autom. Control 19(6), 716-723 (1974).

${ }^{61}$ V. M. R. Muggeo, "Interval estimation for the breakpoint in segmented regression: A smoothed score-based approach," Aust. N. Z. J. Stat. 59, 311-322 (2017).

${ }^{62} \mathrm{~J}$. Paul and S. Narashimhan, "Effect of coordination on bond properties: A first principles study," Bull. Mater. Sci. 31, 569 (2008).

${ }^{63}$ N. Mattern, H. Hermann, S. Roth, J. Sakowski, M.-P. Macht, P. Jovari, and $\mathrm{J}$. Jiang, "Structural behavior of $\mathrm{Pd}_{40} \mathrm{Cu}_{30} \mathrm{Ni}_{10} \mathrm{P}_{20}$ bulk metallic glass below and above the glass transition," Appl. Phys. Lett. 82, 2589 (2003).

${ }^{64} \mathrm{~K}$. Georgarakis, D. V. Louzguine-Luzgin, J. Antonowicz, G. Vaughan, A. R. Yavari, T. Egami, and A. Inoue, "Variations in atomic structural features of a supercooled $\mathrm{Pd}-\mathrm{Ni}-\mathrm{Cu}-\mathrm{P}$ glass forming liquid during in situ vitrification," Acta Mater. 59, 708-716 (2011).

${ }^{65} \mathrm{~K}$. F. Kelton and A. L. Greer, "Transient nucleation effects in glass formation," J. Non-Cryst. Solids 79, 295-309 (1986).

${ }^{66} \mathrm{Y}$. Ashkenazy and R. S. Averback, "Atomic mechanisms controlling crystallization behaviour in metals at deep undercoolings," Europhys. Lett. 79, 26005 (2007).

${ }^{67}$ J. Orava and A. L. Greer, "Fast and slow crystal growth kinetics in glass-forming melts," J. Chem. Phys. 140, 214504 (2014).
${ }^{68}$ E. Kirova and V. Pisarev, "Morphological aspect of crystal nucleation and glass transition in supercooled metallic melt," in 17th International Conference, on Liquid and Amorphous Metals, Lyon, 26-30 August 2019.

${ }^{69} \mathrm{~J}$. W. Christian, The Theory of Transformations in Metals and Alloys (Pergamon Press, Oxford, 1965).

${ }^{70}$ M. T. Clavaguera-Mora, N. Clavaguera, D. Crespo, and T. Pradell, "Crystallisation kinetics and microstructure development in metallic systems," Prog. Mater. Sci. 47, 559-619 (2002).

${ }^{71}$ D. V. Louzguine-Luzgin and A. Inoue, "An extended criterion for estimation of glass-forming ability of metals," J. Mater. Res. 22, 1378-1383 (2007).

${ }^{72}$ J. Lu and J. A. Szpunar, "Applications of the embedded-atom method to glass formation and crystallization of liquid and glass transition-metal nickel," Philos. Mag. A 75, 1057-1066 (1997).

${ }^{73}$ D. V. Louzguine-Luzgin, R. Belosludov, M. Saito, Y. Kawazoe, and A. Inoue, "Glass-transition behavior of Ni: Calculation, prediction, and experiment," J. Appl. Phys. 104, 123529 (2008).

${ }^{74} \mathrm{~W}$. Kauzmann, "The nature of the glassy state and the behavior of liquids at low temperatures," Chem. Rev. 43, 219-256 (1948).

${ }^{75}$ F. J. Cherne, M. I. Baskes, R. B. Schwarz, S. G. Srinivasan, and W. Klein, "Nonclassical nucleation in supercooled nickel," Modell. Simul. Mater. Sci. Eng. 12, 1063-1068 (2004).

${ }^{76}$ L. Zhong, J. Wang, H. Sheng, Z. Zhang, and S. X. Mao, "Formation of monatomic metallic glasses through ultrafast liquid quenching," Nature 512, 177-180 (2014).

${ }^{77}$ J. J. Han, C. P. Wang, X. J. Liu, Y. Wang, Z.-K. Liu, T.-Y. Zhang, and J. Z. Jiang, "Abnormal correlation between phase transformation and cooling rate for pure metals," Sci. Rep. 6, 22391 (2016).

${ }^{78}$ Y. W. Kim, H. M. Lin, and T. F. Kelly, "Amorphous solidification of pure metals in submicron spheres," Acta Metall. 37, 247-255 (1989).

${ }^{79}$ H. A. Davies, "Metallic glass formation," in Amorphous Metallic Alloys, edited by F. E. Luborsky (Butterworths, London, 1983), pp. 8-25.

${ }^{80} \mathrm{O}$. K. Belousov, "Estimation of the critical glass transition rate and the inorganic glass thickness," Russ. Metall. 2009, 488-497.

${ }^{81}$ T. Kulik, "Nanocrystallization of metallic glasses," J. Non-Cryst. Solids $\mathbf{2 8 7}$, 145-161 (2001).

${ }^{82}$ Z. Wang, C. L. Chen, S. V. Ketov, K. Akagi, A. A. Tsarkov, Y. Ikuhara, and D. V. Louzguine-Luzgin, "Local chemical ordering within the incubation period as a trigger for nanocrystallization of a highly supercooled Ti-based liquid," Mater. Design 156, 504-513 (2018).

${ }^{83}$ A. L. Greer, "New horizons for glass formation and stability," Nat. Mater. 14, 542-546 (2015).

${ }^{84} \mathrm{D}$. V. Louzguine-Luzgin and A. Inoue, "Observation of linear defects in $\mathrm{Al}$ particles below $7 \mathrm{~nm}$ in size,” J. Mater. Res. 21, 1347 (2006). 